\title{
VAERS data reveals no increased risk of neuroautoimmune adverse events from COVID-19 vaccines
}

\author{
Chris von Csefalvay ${ }^{1}$ (D)
}

1 Starschema Inc., Arlington, VA; csefalvayk@starschema.net

\begin{abstract}
Neuroautoimmune disorders, such as multiple sclerosis and Guillain-Barre syndrome, have been documented in relation to various vaccines in the past. This paper uses passive reporting information from the CDC/FDA's VAERS system to analyse whether neuroautoimmune presentations are reported at a relatively higher or lower rate, vis-a-vis other adverse effects, for COVID-19 vaccines than for other vaccines. Through computing the reporting odds ratios for a range of symptoms and comparator vaccines, a clear indication in favour of the safety of COVID-19 vaccines emerges, with reports of neuroautoimmune adverse events in relation to other adverse events being over $70 \%$ less likely for COVID-19 than for comparator vaccines ( $R O R: 0.292, p<0.0001$ ). In comparison with other vaccines given as part of routine care in adulthood, COVID-19 vaccines have the lowest reporting odds ratio of neuroautoimmune adverse effects (median ROR: 0.246).
\end{abstract}

Keywords: Neuroautoimmune disorders, demyelinating disorders, vaccine safety, pharmacovigilance, COVID-19 vaccines

\section{Introduction}

The global drive to curb the spread of COVID-19 through a concerted vaccination effort is undoubtedly one of the most ambitious public health projects ever undertaken. At the time of writing, only a little over six months after the FDA granted EUAs to the first two of eventually three SARS-CoV-2 vaccines in early December, over $43 \%$ of the U.S. population has been fully vaccinated (i.e. two doses of a two-dose vaccine or a single dose of a one-dose vaccine). For comparison, during the severe $2017-18 \mathrm{flu}$ season, only $37.1 \%$ of adults were vaccinated.[1]

To safeguard this unprecedented accomplishment, it is crucial to exercise close surveillance of potential adverse events (AEFIs). At least partially owing to the fact that the Moderna and Pfizer/BioNTech vaccines are first-in-man applications of mRNA vaccine technology at scale, a significant number of eligible individuals have refused to be vaccinated.[2-4] The fear of adverse effects, both short and long term, is undoubtedly a driving factor that fuels these apprehensions and eventually results in an overall reluctance to be vaccinated. Among mid- to long-term AEFIs, neurological AEFIs hold a particular place, as is evident from studies on reasons behind vaccine refusal.[5] The bitter past experience with Guillain-Barre syndrome (GBS) as a side effect of certain influenza vaccines, esp. the much publicised association between 1976-77 A/New Jersey/8/76 influenza vaccines and GBS, is still within living memory.[6] Similarly well-known is the association between the recombinant hepatitis B vaccine and multiple sclerosis.[7] In addition, the emergence of various theories about a purported (and disproven) causal mechanism between autism spectrum disorders and vaccinations that hypothesises an autoimmune pathogenetic process has created a persistent point of rhetoric that erroneously portrays vaccines as inevitably likely to cause neuroautoimmunity.[8] Given the often devastating economic, social, psychological and QoL impact of neuroautoimmune diseases,[9-11] some degree of apprehension appears natural.

It is beyond the scope of this paper to outline the range of potential pathophysiological mechanisms that may underlie autoimmunity, including neuroautoimmunity, following a vaccination (for such a review, see Wraith et al. (2003)[12]). Rather, we seek to idenPublisher's Note: MDPI stays neutral with regard to jurisdictional claims in published maps and institutional affiliations. 
(Pfizer/BioNTech, Moderna and Johnson \& Johnson) with regard to neuroautoimmune AEFIs through an analysis of VAERS.

As with all studies leveraging VAERS, its limitations must be read in conjunction with results derived from VAERS data. Like all passive pharmacovigilance systems, it relies on reporting. Under the terms of the EUAs granted to each of the three vaccine manufacturers, COVID-19 vaccination providers must report any

- $\quad$ vaccine administration errors (regardless of consequence);

- $\quad$ serious AEFI (regardless of causal attribution), incl. any AEFI resulting in inpatient admission, long-term disability or death; and

- instances of multi-system inflammatory syndrome,

along with serious immunisation failures (cases of COVID-19 in vaccinated individuals that result in inpatient admission or death). This goes beyond to the typical reporting regime, wherein reporting is encouraged but not mandatory unless the patient was a minor at the time. At the same time, VAERS remains open to reports from individuals, allied healthcare workers and even unrelated third parties.[13-15] In other words, while there is a risk of unreported AEFIs, there may also be a degree of over-reporting (the same AEFI, for instance, may be reported by the physician, the nurse and the patient as well, without the knowledge of any of the other parties). Moreover, VAERS does not verify the accuracy or veracity of reports, nor does it require a causal attribution. This is notably illustrated by the number of accidents, drownings and congenital diagnoses reported to VAERS, none of which could conceivably be a causal consequence of vaccination. Thus, VAERS data must be appropriately analysed to fulfill its function, which is to generate early potential safety signals rather than to substantiate a causal relationship.

In this paper, we are using a reporting odds ratio (ROR) analysis[16] to compare the likelihood that an AEFI reported to VAERS is one of a number of neuroautoimmune AEFIs

between COVID-19 and non-COVID-19 vaccines, concluding that a strong association exists in favour of COVID-19 vaccines. Based on the evidence as it presently stands, the odds of reporting a neuroautoimmune AEFI vis-a-vis any other AEFI are significantly lower than for comparable vaccines, attesting to the safety of COVID-19 vaccines.

\section{Materials and Methods}

Data was obtained for 2015 to 2021, inclusive, from the VAERS website (https: / /vaers. hhs.gov) on 12 June 2021. The data comprises all reports that were received between 01 January 2015 and 28 May 2021, comprising altogether 2,528,763 individual reports. Of these, 1,323,178 (52.33\%) pertained to a COVID-19 vaccine.

\subsection{Categorisation of cases}

Following ingestion using Python v.3.7.5 and pandas v.1.2.4,[17] the resulting data frames were joined and reshaped to yield individual entries per reported symptom. Using the symptom description, reports of the following VAERS symptoms (i.e. symptoms using the coding phraseology as present in VAERS) were coded as involving a neuroautoimmune AEFI:

- Demyelinating polyneuropathy

- Immune-mediated neuropathy

- Axonal neuropathy

- Axonal and demyelinating neuropathy

- Chronic inflammatory demyelinating polyradiculoneuropathy

- Subacute inflammatory demyelinating polyradiculoneuropathy

- Autoimmune neuropathy

- Autonomic neuropathy

- Guillain-Barre syndrome

- Acute disseminated encephalomyelitis

- Demyelinating polyneuropathy 
- Neuromyelitis optica spectrum disorder

- Neuromyelitis optica

- Myelitis transverse

- Multiple sclerosis relapse

- Multiple sclerosis

- Relapsing multiple sclerosis

- Progressive multiple sclerosis

- Relapsing-remitting multiple sclerosis

- Optic neuritis

- Immune-mediated encephalitis

- Autoimmune encephalopathy

- Encephalitis autoimmune

- Autoimmune demyelinating disease

Based on this encoding, the individual data was segmented and age distributions were calculated (Figure 1). Subjects receiving the COVID-19 vaccine were on average older ( $\mu=50.2, \sigma=17.8)$ than those who received other vaccines $(\mu=36.9, \sigma=28.3)$, a phenomenon attributable to the relatively large number of childhood vaccines in relation to vaccines received in later life. Except for male recipients of the COVID-19 vaccine, age distribution of neuroautoimmune disorders followed a bimodal pattern, peaking in the third and eight decades of life. Male recipients of the COVID-19 vaccine who reported a neuroautoimmune AEFI tended towards a single mode in the early seventh decade of life.

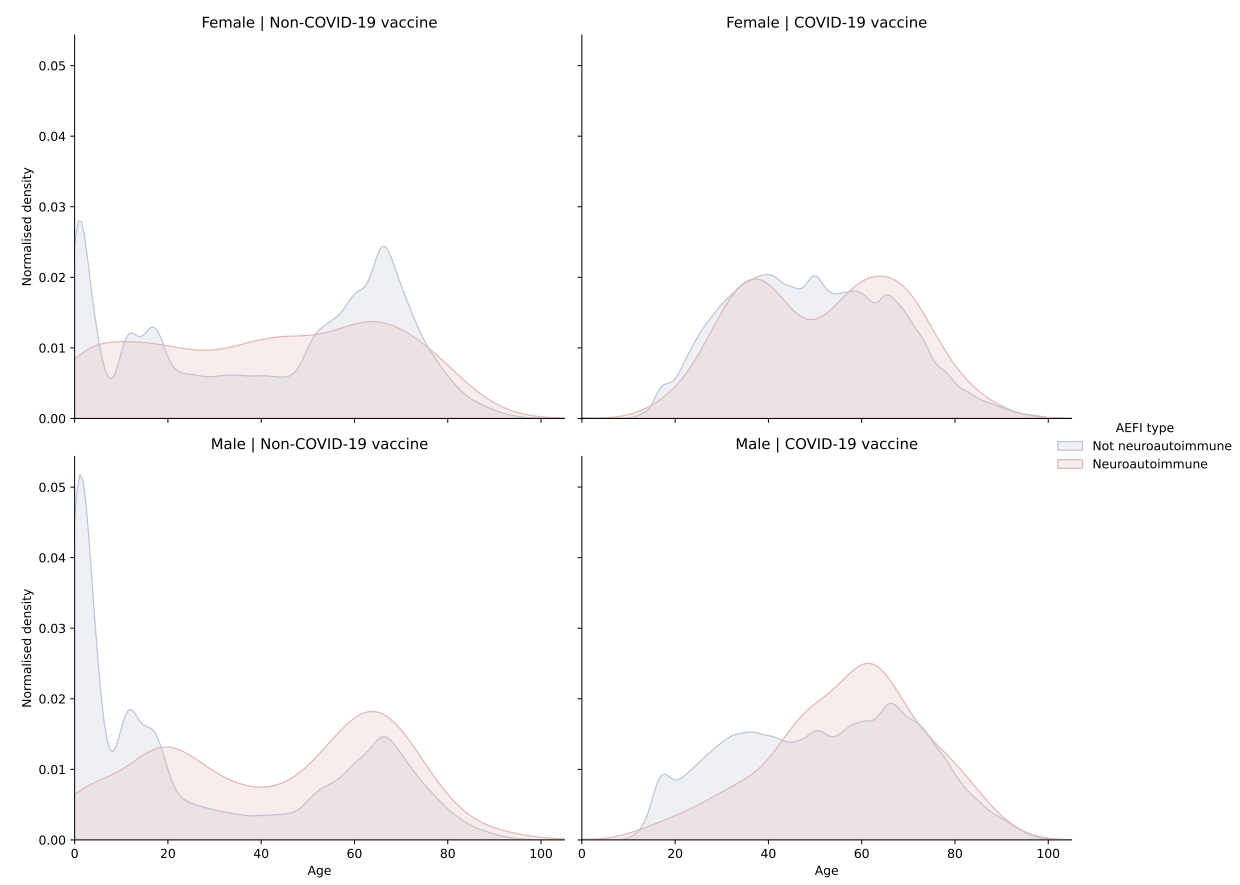

Figure 1. Distribution of reports by age, partitioned by vaccine type (COVID-19 vs. non-COVID-19 vaccine) and neuroautoimmune disorder status (neuroautoimmune vs. non-neuroautoimmune symptoms).

Data was cross-tabulated to yield a 2x2 contingency table for COVID-19 vs. nonCOVID-19 vaccines (Table 1), and the odds ratio was calculated using Fisher's exact test. In addition, a Pearson's $\chi^{2}$ test was performed and the Pearson residuals were calculated for each permutation, using statsmodels v.0.12.2.[18] 
Table 1. $2 \times 2$ contingency table by vaccine type (COVID-19 vs. non-COVID-19 vaccine) and neuroautoimmune disorder status.

\begin{tabular}{ccc}
\hline & Neuroautoimmune AEFI & Non-neuroautoimmune AEFI \\
\hline COVID-19 vaccine & 612 & $1,322,566$ \\
Non-COVID-19 vaccine & 1908 & $1,203,677$ \\
\hline
\end{tabular}

Next, symptoms were broken down individually, and a $\chi^{2}$ distribution fitted. Along with the actual values, the expected values under the null hypothesis of independence $\left(H_{0}\right)$ were ascertained, as well as the Pearson residuals.

Finally, a large contingency table was constructed, calculating the reporting odds ratio of each AEFI for each vaccine category (VAERS variable VAX_TYPE), which acts as a superset comprising vaccines that have typically the same target and same valency (e.g. the Johnson \& Johnson COVID-19 vaccine and the Moderna vaccine are both in the COVID19 vaccine category, whereas a trivalent and a quadrivalent influenza vaccine would be different vaccine categories due to their difference in valency). For the purposes of constructing this table, a regular expression was used to filter out a number of entities that are typically recorded in VAERS but which do not indicate an appropriate denominator for the calculation of the odds ratio. These were used to exclude

- normal results (e.g. .*(negative|normal)\$),

- mere tests and assays that do not disclose a result (e.g. .*assay\$),

- procedures (e.g. .*(plasty|insertion|tomylery...),

- management of an ongoing medical status or device,

- administrative flags (e.g. Blood (group/don (or / ation))), and

- COVID-19 related public health interventions (e.g. COVID-19

(prophylaxis|immunisation/screening)).

RORs were cross-tabulated by vaccine type and filtered only for symptoms that were categorised as neuroautoimmmune symptomatic presentations.

\section{Results}

\subsection{Association with neuroautoimmune AEFIs}

Fisher's two-sided exact test was used to calculate the odds ratio to determine the odds ratio for an association between reporting a neuroautoimmune AEFI and reporting after/in relationship with a COVID-19 vaccine. This yielded an OR of 0.292 at $p<0.0001$ - a statistically highly significant result that indicates COVID-19 vaccination predisposes to a significantly lower risk - by over $70 \%$ - of reporting a neuroautoimmune side effect rather than any other side effect. It is important to note that this does not mean that neuroautoimmune side effects are $70 \%$ less frequent, but rather that the likelihood of reporting a neuroautoimmune side effect over any other side effect is $70 \%$ lower.

Table 2. Pearson residuals by vaccine type (COVID-19 vs. non-COVID-19 vaccine) and neuroautoimmune disorder status.

\begin{tabular}{ccc}
\hline & Neuroautoimmune AEFI & Non-neuroautoimmune AEFI \\
\hline COVID-19 vaccine & -19.459 & 0.615 \\
Non-COVID-19 vaccine & 20.386 & -0.644 \\
\hline
\end{tabular}

This is confirmed by the Pearson $\chi^{2}$ residuals that were calculated (see Table 2). The residual of neuroautoimmune AEFIs following a COVID-19 vaccine $(-19.459)$ indicates that the expected value is significantly higher than the actual value under the assumption of the null hypothesis of independence. It is therefore quite evident that the odds of reporting a neuroautoimmune AEFI over any other AEFI are correlated with whether the 
preceding vaccine was a COVID-19 vaccine or not, with recipients of COVID-19 vaccines being significantly $(70 \%)$ less likely to report a neuroautoimmune AEFI.

\subsection{Association of individual neuroautoimmune AEFIs}

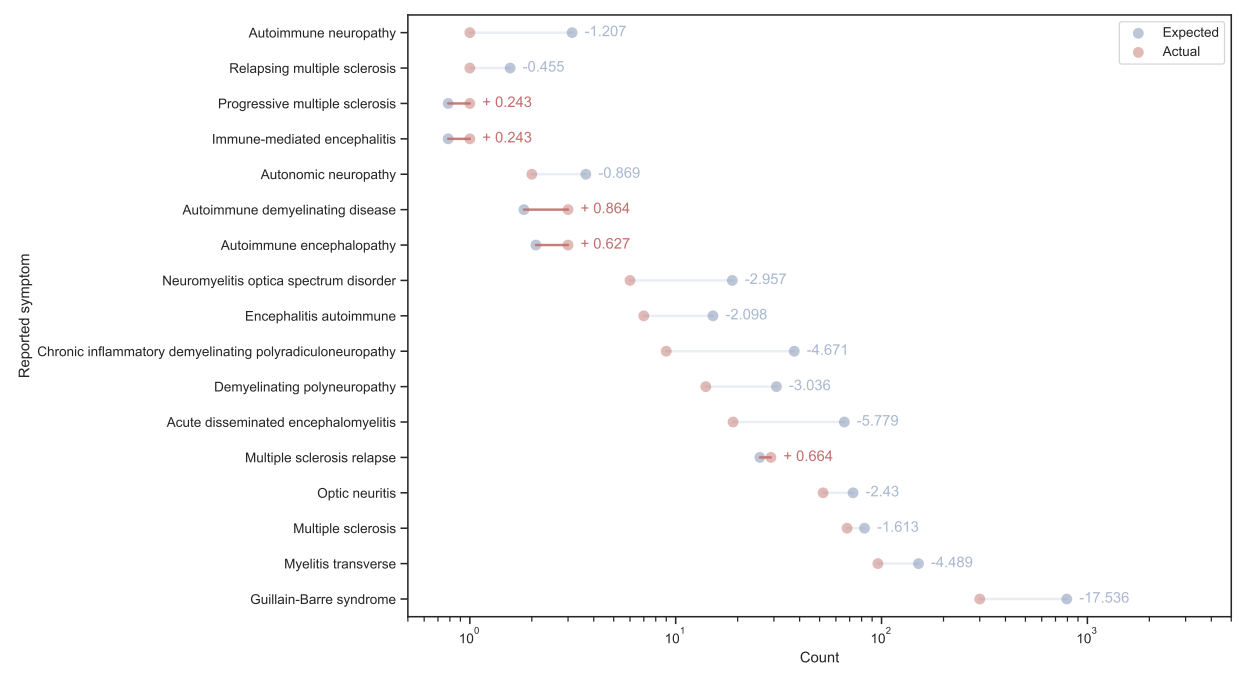

Figure 2. Expected vs. actual number of reports for individual neuroautoimmune symptoms based on Pearson's $\chi^{2}$ test along with Pearson residuals for COVID-19 vaccines.

Following on from these findings, it is not surprising to see that in most cases, the number of actual cases reported is significantly lower than would be expected under the null hypothesis of independence (see Figure 2). The notable exceptions are

- progressive MS (Pearson residual: 0.243),

- immune-mediated encephalitis (Pearson residual: 0.243),

- autoimmune demyelinating disease (Pearson residual: 0.864),

- autoimmune encephalopathy (Pearson residual: 0.627), and

- $\quad$ MS relapse (Pearson residual: 0.664).

While the residuals indicate that the deviation is quite small for all of the above, it appears that in contrast with other vaccines, COVID-19 vaccines appear to result in a relatively higher number than expected of these AEFIs. This must be considered in the context of actual figures, of course - altogether, these differences account for fewer than 0.25 additional cases of progressive MS and immune-mediated encephalitis, fewer than one case of autoimmune encephalopathy, fewer than 4 cases of MS relapse and fewer than 1.5 cases of autoimmune demyelinating disease. Of over $1.3 \mathrm{~m}$ reports, these constitute an excess of six cases, or approx. one in 220,530 reports. Consequently, it remains to be seen (especially given the low absolute numbers) whether this association remains in evidence as more data accrues, or, as is more likely, it is part of the noisy undercurrent that is characteristic of passive reporting. 


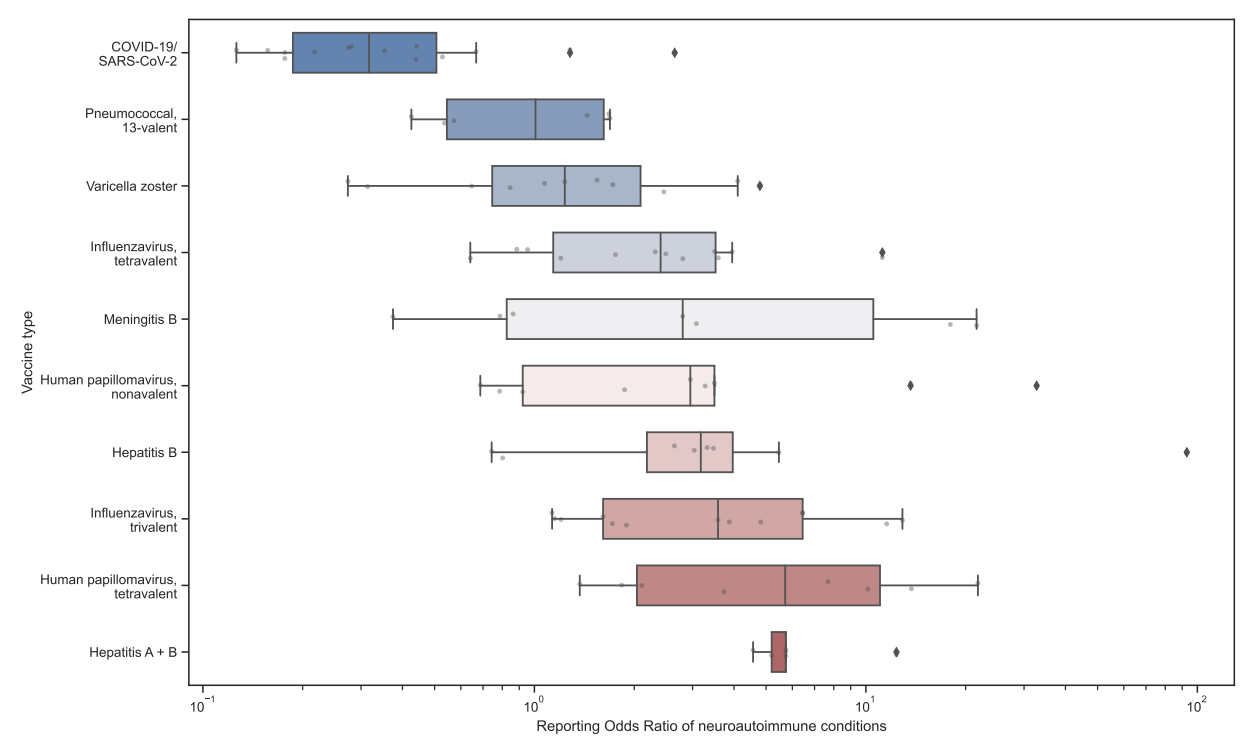

Figure 3. Reporting Odds Ratios of neuroautoimmune conditions in a range of vaccines approved for adult populations.

As Figure 3 shows, the ROR of neuroautoimmune presentations is significantly lower for COVID-19 vaccines than it is for any of the comparator vaccines. The group of comparators was chosen to primarily include vaccines that have the same age range of approved users to reduce the bias arising from the fact that some vaccines are typically given in a paediatric context (e.g. MMR), while COVID-19 vaccination was consciously designed to address older populations first due to their higher overall risk from COVID-19 as well as the relatively higher rate of respiratory, cardiac and endocrine comorbidities that governed vaccine allocation in the beginning.

\section{Discussion}

Based on early data from VAERS for reports about COVID-19 vaccinations received on or before 28 May 2021, there is convincing evidence that COVID-19 vaccines compare remarkably well with other vaccines in respect of neuroautoimmune AEFIs. Despite the limitations of this study, which drew on passive reporting data with all its inherent problems, such as reporting bias and the lack of a reliable denominator, we have found evidence that supports the safety of COVID-19 vaccines with regard to neuroimmunological presentations. Statistical analysis indicated that in comparison to recipients of other vaccines, those who reported in respect of a COVID-19 vaccines were over 70\% less likely to report a neuroimmunological presentation than a non-neuroimmunological presentation.

Similarly, the number of reports fell significantly under what was expected in all but two somemwhat cohesive symptom groups: relapses of MS on one hand and autoimmmune encephalitis on the other. The fact that some forms of autoimmune encephalitis are distributed bimodally by age with a peak in the third and the seventh decade of life may support a hypothesis that the increase in cases of autoimmune encephalitis is the consequence of statistical noise and/or sampling bias due to the higher average age of COVID-19 vaccine recipients in the sample.[19] Similarly, multiple sclerosis exhibits a similar bimodality of age distribution, to the point that respective terms for early-onset (teens to early 30s) and late onset (50s and later) MS have become established in the literature.[20] Nevertheless, clinicians may, as a matter of abundance of caution, keep a closer eye on patients with known MS or risk factors for MS (such as previous diagnosis of optic neuritis or CIS/RIS) for signs of progression or relapse. This may be accompanied by proactively 
explaining the relative risk of a relapse in the context of potentially worse clinical outcomes with COVID-19, especially for patients treated with systemic immunosupressants.

Since the start of the global vaccination campaign, evidence has been accumulating for the overall tolerability and safety of the COVID-19 vaccines. This study seeks to add to our current understanding by quantifying the highly favourable risk profile of COVID19 vaccines with regard to neuroautoimmune disorders. While some questions remain open and conclusions made over early data may have to be reconfirmed through ongoing analysis, the current data supports the assertion that the COVID-19 vaccine has perhaps the lowest reporting odds ratio for neuroautoimmune disorders of any vaccine routinely given in adulthood. This is a reassuring indication of safety at a time when concerns about adverse events still keep most countries from attaining the threshold of collective immunity.

Funding: This research was funded by Starschema Inc. under its intramural research funding programme.

Data Availability Statement: VAERS reporting data is available from the CDC's website at https: //vaers.hhs.gov. All code and scripts supporting this manuscript are deposited at https:/ / github. com/chrisvoncsefalvay/covid19-neuroautoimmune-aefis and are made available under the DOI 10.5281 /zenodo.4940261.

Conflicts of Interest: $\mathrm{CvC}$ is a consultant to a company that may be affected by the research reported in this paper. The funders had no role in the design of the study; in the collection, analyses, or interpretation of data; in the writing of the manuscript, or in the decision to publish the results.

\author{
Abbreviations \\ The following abbreviations are used in this manuscript: \\ AEFI Adverse event following immmunization \\ CDC Centers for Disease Control and Prevention \\ CIS clinically isolated syndrome \\ EUA Emergency Use Authorization \\ FDA Food and Drug Administration \\ GBS Guillain-Barre syndrome \\ mRNA messenger RNA \\ MS multiple sclerosis \\ QoL quality of life \\ RIS radiologically isolated syndrome \\ ROR Reporting Odds Ratio \\ VAERS Vaccine Adverse Effect Reporting System
}

\title{
References
}

1. CDC. Estimates of influenza vaccination coverage among adults-United States, 2017-18 flu season. Retrieved October 2018, 25, 2018.

2. Dror, A.A.; Eisenbach, N.; Taiber, S.; Morozov, N.G.; Mizrachi, M.; Zigron, A.; Srouji, S.; Sela, E. Vaccine hesitancy: the next challenge in the fight against COVID-19. European Journal of Epidemiology 2020, 35, 775-779.

3. Robertson, E.; Reeve, K.S.; Niedzwiedz, C.L.; Moore, J.; Blake, M.; Green, M.; Katikireddi, S.V.; Benzeval, M.J. Predictors of COVID-19 vaccine hesitancy in the UK household longitudinal study. Brain, Behavior, and Immunity 2021, 94, 41-50.

4. Troiano, G.; Nardi, A. Vaccine hesitancy in the era of COVID-19. Public Health 2021.

5. Berry, S.D.; Johnson, K.S.; Myles, L.; Herndon, L.; Montoya, A.; Fashaw, S.; Gifford, D. Lessons learned from frontline skilled nursing facility staff regarding COVID-19 vaccine hesitancy. Journal of the American Geriatrics Society 2021.

6. Haber, P.; DeStefano, F.; Angulo, F.J.; Iskander, J.; Shadomy, S.V.; Weintraub, E.; Chen, R.T. Guillain-Barré syndrome following influenza vaccination. JAMA 2004, 292, 2478-2481.

7. Hernán, M.A.; Jick, S.S.; Olek, M.J.; Jick, H. Recombinant hepatitis B vaccine and the risk of multiple sclerosis: a prospective study. Neurology 2004, 63, 838-842. 
8. Poland, G.A.; Jacobson, R.M. Understanding those who do not understand: a brief review of the anti-vaccine movement. Vaccine 2001, 19, 2440-2445.

9. Marck, C.H.; Aitken, Z.; Simpson, S.; Weiland, T.J.; Kavanagh, A.; Jelinek, G.A. Predictors of change in employment status and associations with quality of life: a prospective international study of people with multiple sclerosis. Journal of Occupational Rehabilitation 2020, 30, 105-114.

10. Miller, A.; Dishon, S. Health-related quality of life in multiple sclerosis: the impact of disability, gender and employment status. Quality of Life Research 2006, 15, 259-271.

11. Patwardhan, M.; Matchar, D.; Samsa, G.; McCrory, D.; Williams, R.; Li, T. Cost of multiple sclerosis by level of disability: a review of literature. Multiple Sclerosis Journal 2005, 11, 232-239.

12. Wraith, D.C.; Goldman, M.; Lambert, P.H. Vaccination and autoimmune disease: what is the evidence? The Lancet 2003, 362, 1659-1666.

13. Chen, R.T.; Rastogi, S.C.; Mullen, J.R.; Hayes, S.W.; Cochi, S.L.; Donlon, J.A.; Wassilak, S.G. The Vaccine Adverse Event Reporting System (VAERS). Vaccine 1994, 12, 542-550.

14. Shimabukuro, T.T.; Nguyen, M.; Martin, D.; DeStefano, F. Safety monitoring in the vaccine adverse event reporting system (VAERS). Vaccine 2015, 33, 4398-4405.

15. Singleton, J.A.; Lloyd, J.C.; Mootrey, G.T.; Salive, M.E.; Chen, R.T.; Ellenberg, S.; Rastogi, S.; Krueger, C.; Braun, M.; Wise, R.; others. An overview of the vaccine adverse event reporting system (VAERS) as a surveillance system. Vaccine 1999, 17, 2908-2917.

16. Rothman, K.J.; Lanes, S.; Sacks, S.T. The reporting odds ratio and its advantages over the proportional reporting ratio. Pharmacoepidemiology and Drug Safety 2004, 13, 519-523.

17. McKinney, W.; others. pandas: a foundational Python library for data analysis and statistics. Python for High Performance and Scientific Computing 2011, 14, 1-9.

18. Seabold, S.; Perktold, J. Statsmodels: Econometric and statistical modeling with python Proceedings of the 9th Python in Science Conference. Austin, TX, 2010, Vol. 57, p. 61.

19. Shan, W.; Yang, H.; Wang, Q. Neuronal Surface Antibody-Medicated Autoimmune Encephalitis (Limbic Encephalitis) in China: A Multiple-Center, Retrospective Study. Frontiers in Immunology 2021, 12, 344 .

20. Kis, B.; Rumberg, B.; Berlit, P. Clinical characteristics of patients with late-onset multiple sclerosis. Journal of Neurology 2008, 255, 697-702. 
medRxiv preprint doi: https://doi.org/10.1101/2021.06.13.21258851; this version posted June 17, 2021. The copyright holder for this preprint (which was not certified by peer review) is the author/funder, who has granted medRxiv a license to display the preprint in perpetuity. It is made available under a CC-BY 4.0 International license. 

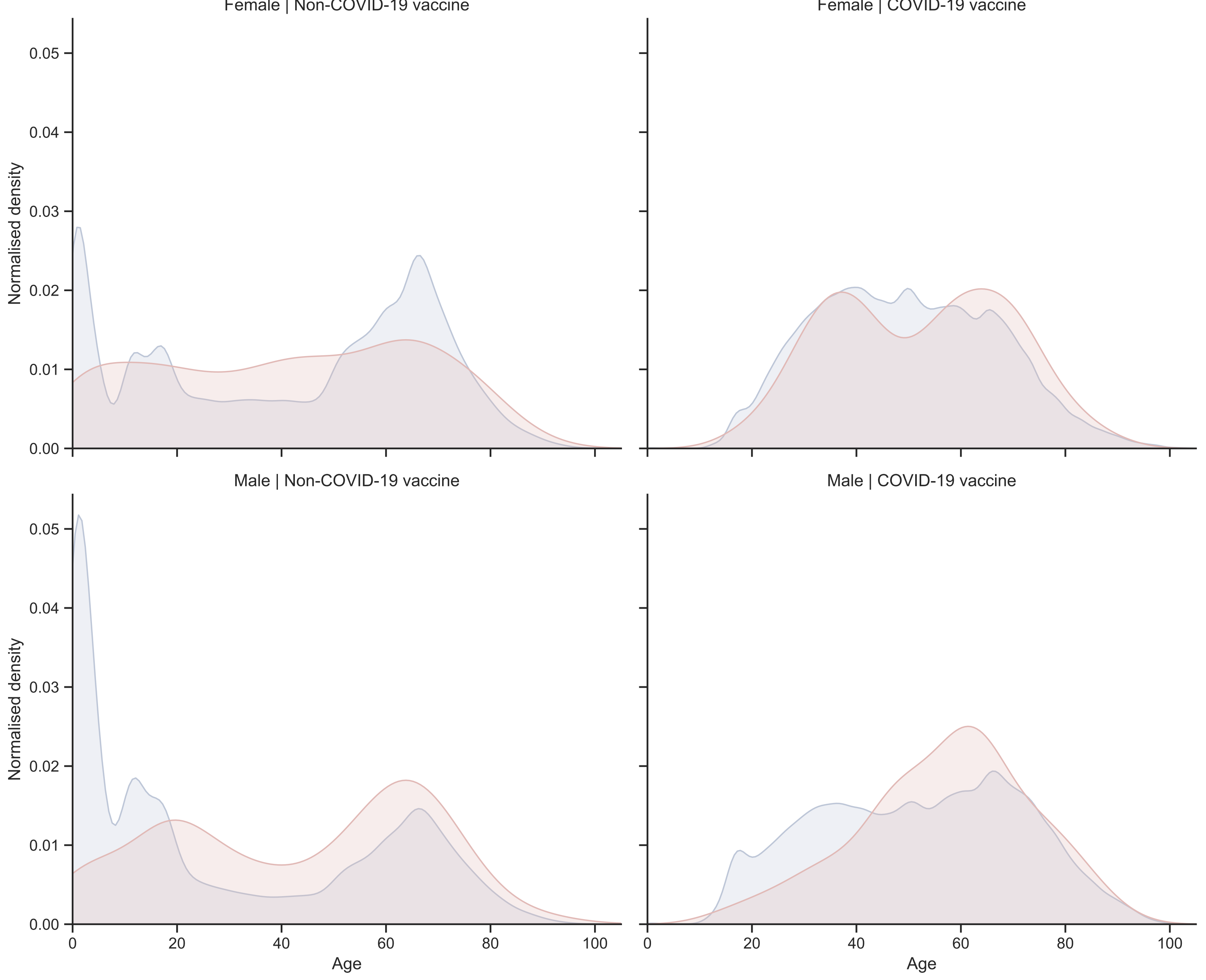

AEFI type

Not neuroautoimmune Neuroautoimmune 


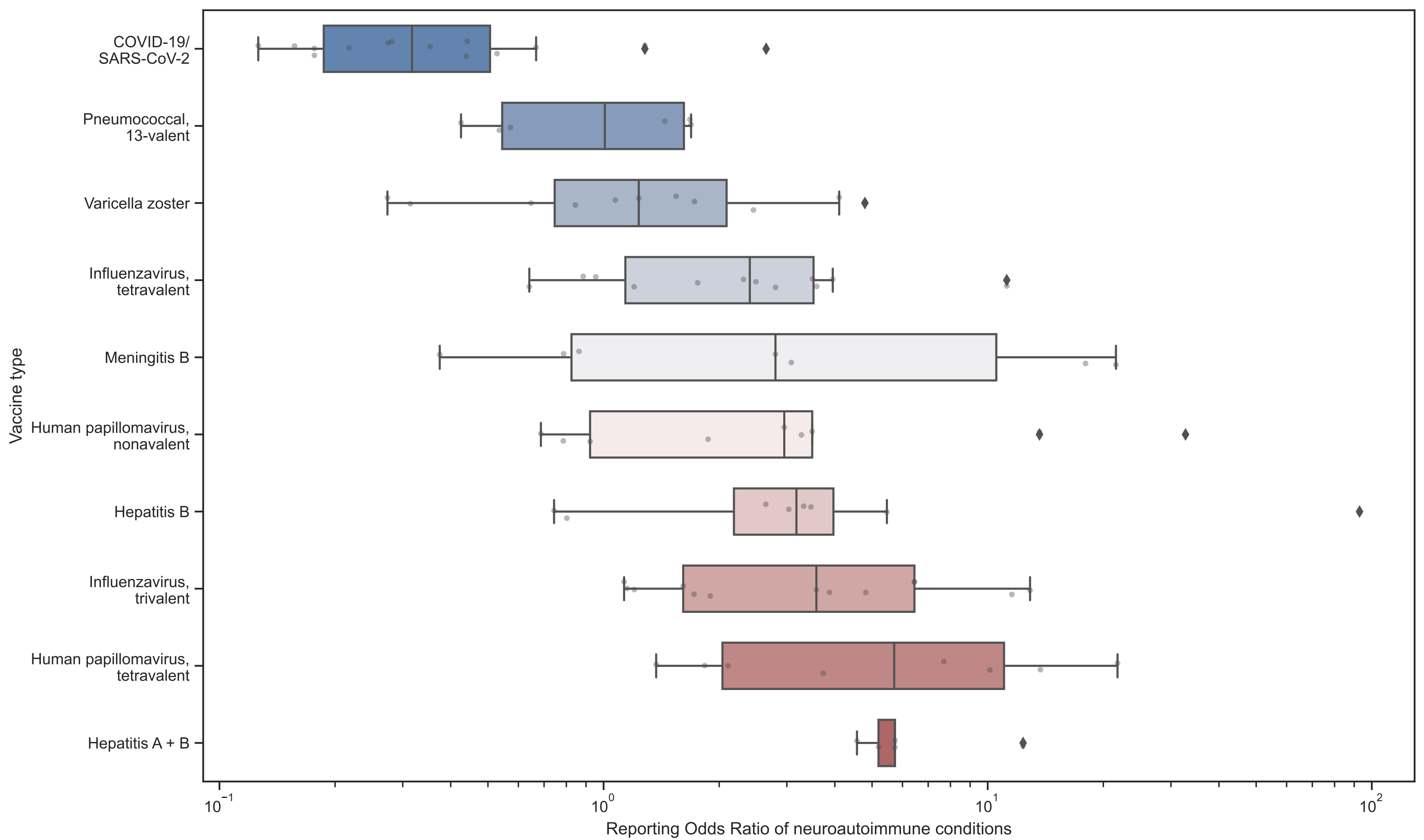

